\title{
Evolution of a Technology Standard Alliance Based on an Echo Model Developed through Complex Adaptive System Theory
}

\author{
Hong Jiang $\mathbb{D},{ }^{1}$ Chen Chen $\mathbb{D}^{1},{ }^{1}$ Shukuan Zhao $\mathbb{D},{ }^{1}$ and Yuhao $\mathrm{Wu} \mathbb{D}^{1,2}$ \\ ${ }^{1}$ School of Management, Jilin University, Changchun, Jilin 130022, China \\ ${ }^{2}$ Rotterdam School of Management, Erasmus University, Rotterdam 3062 PA, Netherlands \\ Correspondence should be addressed to Shukuan Zhao; zhaosk@jlu.edu.cn
}

Received 6 August 2020; Revised 8 October 2020; Accepted 10 November 2020; Published 23 November 2020

Academic Editor: Yi Su

Copyright (c) 2020 Hong Jiang et al. This is an open access article distributed under the Creative Commons Attribution License, which permits unrestricted use, distribution, and reproduction in any medium, provided the original work is properly cited.

The evolution of the technology standard alliance (TSA) is examined using complex adaptive system (CAS) theory. Taking TSA as a dynamic CAS, an echo model is constructed to depict the mechanism of its evolution, and a model is simulated on the NetLogo platform. The echo model includes a basic model, an extended model, and a three-layer echo model. The adhesive aggregation of agents is explained, and the three evolutionary stages of agents' entry, migration, and exit are analyzed. Moreover, the adaptability of agents in TSA is quantified. The results of simulation show the evolution of the TSA in relation to the two aspects of agent adhesion aggregation and agent resource interaction, and they demonstrate the dynamic and complex hierarchical structure of the TSA system. It is proposed that greater matching ability, moderate behavior income, and lower behavior cost are more conducive to the evolution and development of TSA. Additionally, the echo model is reconstructed to expand the range of application of CAS theory.

\section{Introduction}

As science and technology have developed, technology standards have become a strategic command point for enterprise competition, and they affect enterprise behavior. Many enterprises actively participate in and even dominate the formulation of certain international technology standards to take the initiative in the formulation of standards and, through a novel approach to competition regarding them, to establish and maintain their core competitive advantages $[1,2]$. A technology standard is a set of specifications that all elements of a product, process, format, or procedure within its jurisdiction must conform to [3]. Standards are of great strategic significance and can promote future development of enterprises, industries, and countries [4]. The present high degree of complexity of technology, the high risk and high cost of technological development, and the rapid shortening of the product life cycle restrict any single enterprise from providing all of the resources and technologies needed for R\&D and the promotion of technology standards [5]. Therefore, the development of technology standards must be carried out together with other enterprises or organizations [6] to enhance their core competitiveness and help maintain long-term competitive advantages [7].

A technology standard alliance (TSA) is a kind of strategic alliance that arises to support enterprises as they formulate technology standards for a technology. The concept was first put forward formally in the 1990s [8]. A TSA is a typical alliance portfolio [9] or alliance network form [10], which takes a core of enterprises with strong R\&D strengths and key technology intellectual property rights and unites others to it to jointly launch and spread a technology standard in a market [11]. TSAs are widely adopted because they can avoid reusing of resources, distribute enterprise risks, reduce costs, eliminate user concerns, and create a first-mover advantage [12]. Research on TSAs mostly focuses on the concept characteristics $[13,14]$, influencing factors [15, 16], mechanisms of operation [17], and performance evaluation [18]. At present, there have been few studies of the evolution of the TSA, and most of have focused on knowledge ecology [19] and innovation level [20]. Few 
have studied the TSA from a system perspective. The TSA phenomenon is complex, because any evolution of standards involves both macro (environmental) and micro (firm) forces and because standards both drive and are driven by the actions of firms and/or industry associations [21, 22].

In fact, the evolution of the TSA is a complex process and is the result of multilevel co-evolution among alliance agents on the one hand and between alliance agents and the external environment on the other. The interactions among agents and between agents and the environment are complex, and their specific mechanisms are not clear. The theory of complex adaptive system (CAS) focuses on the interaction of major factors within the system, and it highlights overall global behavior through interaction and feedback between the local model and the global model [23]. Using the CAS theory to build a model can provide solutions for dealing with the problems of complex systems. Kauffman [24] introduced CAS theory into organizational research. Warfield [25] studied complex aspects of organizational management and proposed a method of interactive management to solve complex problems in organizational management. Babaoglu et al. [26] found that adaptive agents in society travel through peer-to-peer networks and solve complex problems through node interaction and cooperation with other nodes. The application of CAS theory focuses on industrial clusters, ecosystems, or specific organizational structures. However, the TSA is a new type of strategic alliance, and there has been little application of CAS theory to study of TSAs. In addition, insufficient attention has been paid to the number of agents in a TSA setup, and previous models do not reflect interaction between agents and the environment. Therefore, it is necessary to apply CAS theory to create a global model to comprehensively and systematically investigate the evolution of the TSA.

The entire performance of TSA is a comprehensive embodiment of the behavior of the agents within the alliance. This paper takes the TSA as the research object, constructs a local model of internal agents, and then constructs a global model of the TSA, including the environmental factors. To accomplish our objective, we first analyze the complex adaptive characteristics of the TSA system and demonstrate that it is a typical CAS. Next, we build local models of agent interaction and a three-layer echo model that is suitable for the whole alliance, based on the idea of echo. Then, through simulation analysis of the evolutionary process of the TSA, we obtain the key factors that are conducive to the evolution and development of the alliance. Finally, we discuss and summarize the major managerial implications of our findings.

\section{Summary of the Theory}

CAS theory was put forward by Professor Holland [27] on the 10th anniversary of the founding of the Santa Fe Institute. Its core idea is that adaptation builds complexity. It is the foundation for an important branch of complexity science [28], which introduces the concept of agent with the adaptive ability to recognize and describe the behavior of a complex system in relation to the interaction between the agents and environment [29]. A CAS is invariably composed of a large number of active agents, called adaptive agents. As they accumulate experience, they adapt to change by constantly changing their rules [30]. In a CAS, all agents appear in a common environment, but each one conducts adaptive learning and evolution in parallel and independently, according to the local small environment around it [31]. The main part of the environment of any particular adaptive agent is composed of other adaptive agents. Therefore, each agent becomes adapted to the other adaptive agents.

The CAS operates on both the macroscopic and microscopic levels. At the macro level, it focuses on the hierarchy, diversity, and aggregation of agents [32] and emphasizes the interaction between them and the environment, so that a system that is composed of the agents is constantly evolving, exhibiting a range of complex evolutionary processes, such as emergence [33]. At the microlevel, the initiative and adaptability of the agent are emphasized. The agent actively learns or accumulates experience and changes its behavioral mode to adapt to changes. The evolution of the whole system is gradually derived from the agent [34]. In CAS theory as constructed by Holland, seven basic points are seen [27]. These are the common points extracted from all CASs, and other common points can be derived from a combination of these. They refer to the four characteristics and three mechanisms found in all CASs, namely, aggregation, nonlinearity, flows, diversity, tagging, internal model, and building blocks. The first four are some attributes of the agents, which play a role in adaptation and evolution, and the later three are the mechanism of interaction between the agents and environment. At the same time, these seven basic points also define the idea of a CAS from another angle. That is, a system with these characteristics can be understood as a CAS.

In relation to the behavior and interaction of adaptive agents, Holland proposed a macromodel of CAS, namely Echo. The Echo model is based on resources and location, and it ranges from simple to complex. Resources refer to any environmental material which affects the survival and development of the agents. The foundation of the echo model is laid by a series of renewable resources, and some resources will be consumed or used in the system, which maintains the activities of the agents with active adaptability. Location is the space place of the agent activity in a CAS. Each agent is located in different geographical positions or different development space, where the abundance of resources is also distinct. Because of the interaction between the agents, the geographical environment of the echo model is constituted by a group of interconnected locations. In the basic model, the agent is composed features attack tag, defense tag, and resource database [35]. In this model, the future of a given agent depends entirely on the tagging pairs that it carries. The resources obtained by an agent are directly proportional to the degree to which its attack tag matches other agents' defense tags. However, the basic model cannot fully describe other emergent phenomena of CASs, so the five mechanisms of conditional exchange, resource transformation, adhesion, selective mating, and conditional replication are gradually extended [36]. Through the gradual expansion, the expressive 
and descriptive ability of echo model is enhanced, which enables it to describe and study various complex systems. Holland's echo model draws on multidisciplinary knowledge theory to depict the ways in which CASs evolve, adapt, condense, compete, and cooperate. Thus, the echo model is an extremely beautiful model, constructed from very few principles, that provides a roadmap for how complexity emerges and adapts [37]. Nevertheless, in social science, it is difficult to perform a quantitative analysis of system complexity with objective data, especially for finding or designing feasible methods of calculation in the echo model. Therefore, this paper applies CAS theory to the TSA context and draws lessons from the basic idea of the echo model to design the quantitative method of agents' adaptability and the mechanism of alliance evolution.

\section{Complex Adaptive Characteristics of the TSA System}

The enterprise itself is a complex system, but the TSA, which has the enterprise as the main agent, shows even more complexity. As an adaptive agent, enterprises in an alliance interact with other agents and the environment of the alliance, leading to the performance of a series of complex behaviors, which will lead to the evolution and development of TSA. This paper takes the TSA as its research object, with an approach based on CAS theory. In other words, the TSA is seen to have seven basic points. According to CAS theory, the complex system of the TSA can be simplified by abstracting the key points out from the system. Therefore, we analyze the complex characteristics of the TSA system to simplify it, and we verify the hypothesis that the TSA is a CAS. This provides an occasion to analyze the adaptive behavior of the alliance agents and provides ideas for further research on the evolution and development of the TSA.

3.1. Aggregation. In CAS analysis, aggregation has two meanings. First, it is a means of simplifying complex systems. It refers to the aggregation of similar things into classes, which is the basis of model construction. The second meaning is more relevant to the content of CAS, which refers to simple agents that are gathered together to form a highly adaptive aggregate, also known as a higher level of metaagent. Their interaction can produce more complex emergent phenomena, and the overall benefits may exceed the sum of the benefits for each agent, respectively.

The TSA is a contractual alliance organization used to jointly carry out standardization activities, formulating, implementing, and diffusing alliance technology standards, with enterprises as the main participants [38]. To realize their own development goals, these enterprises and organizations gather and form a TSA, which produces a series of spontaneous and complex behaviors, promotes the industrialization of the development of standards, and facilitates cost saving, interconnection, module innovation, and system integration, based on a common interface of related products [39]. Moreover, the TSA is formed by enterprises with strong R\&D strength and key intellectual property rights for technology as the core, combining multiple enterprises or organizations. It is a self-organized, dynamic formation, which can easily cause emergent phenomena [40]. The boundary of the alliance then expands with the addition of new agents, and it then produces aggregation on multiple levels. Other agents and the alliance system as a whole respond, and adaptability and alliance performance change.

3.2. Nonlinearity. When agents and their attributes change in the TSA system, they no longer follow a simple linear relationship, instead showing nonlinear characteristics, especially in relation to repeated interactions with the system or environment [41]. In internal and external interactions in the TSA system, various complex relationships appear, which inevitably form the nonlinear characteristics of the TSA [42]. Specifically, in relation to the overall development of the TSA, network density [43], structural wholes [44], the small world effect [45], relationship strength [46], embeddedness [47], and government intervention [48] have an impact on the TSA, and partner selection $[49,50]$ and network centrality [51] are the main influencing factors for enterprise development in this context. There are many uncertain and fuzzy influencing factors in the external environment of the TSA system, and each agent must strengthen its own competitiveness by learning to adapt to the environment, thus making the nonlinear characteristics of the TSA system more obvious [52]. Generally speaking, complex relationships among agents, between the agents and the system, and between the system and the external environment can lead to nonlinear behavior and results and increase the uncertainty and possibility of the system's predictions.

3.3. Flows. In the TSA system, flows of information, materials, energy, and other resources appear between the agents and the environment. These flows vary from time to time and may even disappear as agents adapt or show maladaptation, and they have multiplier and recycling effects as well. Through cooperation and the sharing and diffusion of new knowledge, alliance members can accelerate the speed of knowledge innovation, and the knowledge level of the whole alliance can be improved due to this flow $[53,54]$. As resources become invested in a certain agent, the effect produced by that agent is called the initial effect; then, resources are transferred from one agent to another in various forms, resulting in a series of changes. The total effect generated by this transfer flow multiplies the initial effect; that is, the TSA system produces a multiplier effect. Moreover, after resources from a certain agent complete their flow, they flow back to the agent, and this recycling flow produces additional resources for each agent that is connected to resources, causing a recycling effect. In addition to knowledge flow, resource flows such as information flow, capital flow, energy flow, and technology flow also exist. Each agent in the TSA system must exchange resources with the environment or other agents to survive and develop. An agent is connected with others to enable resource flow, which is an important motivation for the formation of TSA 
[55]. The reason that the TSA continuously evolves, functions, and creates value is inseparable from the continuous flow of various resources between the agents of the alliance and the external environment.

3.4. Diversity. The diversity of CASs is the result of continuous adaptation. Each agent is located in a niche that is defined by an interaction centered on that agent. When an agent changes, it moves to another niche and a vacancy is generated. The system makes a series of adaptive responses, generates a new agent to fill the empty niche, and provides most of the lost interactions. Obviously, a TSA also has diversity as a characteristic, which makes the research of TSA more complex. The following discusses the diversity of a TSA in relation to agent diversity, technological diversity, and geographical diversity [56].

3.4.1. Agent Diversity. In addition to technology enterprises that have R\&D functions and production functions, the members of a TSA may also be governments, universities, scientific research institutions, and industry associations [57], and these include organizations in the upper, middle, and lower reaches $[58,59]$.

3.4.2. Technological Diversity. In a TSA system, resource diversity can be considered in relation to technology. Technological diversity intuitively reflects the degree of focus and dispersion of different technological categories [60]. The technology owned by each agent in the alliance is diversified, so the TSA system also features technological diversity. Moreover, as new agents are added and the alliance evolves and develops, technological diversity increases accordingly [61].

3.4.3. Geographical Diversity. This means the degree of cultural, economic, and institutional differences [62]. The geographical location of each agent in the TSA system differs, and they may be located in different cities or countries [63]. For this reason, the management theories and cultural systems of different agents are also different. Some scholars use indexes of cultural or institutional distance to measure the level of difference level in the alliance combination [64].

In addition, different functions, such as market positioning [65], governance mode, and exploration/utilization [66], also bring diversity to the TSA. In essence, these diversities are caused by different configurations of the TSA. In a word, the configuration, alliance structure, management mode, standardization capability, and environment of a TSA system [67] are affected by many factors and they have an impact on the system in turn. Therefore, the TSA system presents the characteristics of diversity.

3.5. Tagging. Tagging is a mechanism that guides common hierarchical organizational structures in a CAS. In brief, it distinguishes and selects an agent or target by using the tagging. The interaction and aggregation of the agents based on tagging forms the TSA system, and from it, meta-agents and organizational structures emerge.

For agents in a TSA, organizational scale, market position, product advantage, resource capability, and geographical environment are the main tags for the choice of cooperation. Tagging has a particularly important role to play in selecting alliance partners and building and managing alliance networks [68]. The agent can choose alliance partners based on market orientation, with certain information or resources as the tag, and it can choose alliance partners that have scarce resources that are difficult to imitate and vital for improving their own ability to promote innovation and economic performance through effective integration with the internal resources of the agents [69]. At the same time, sales volume, operating cost, technology exchange and learning, developmental risk, and other aspects [70] can also be used as tags for the TSA system. The existence of these tags is conducive to the selection and cooperation of the agents in a TSA, the smooth development and realization of agents' own goals and tasks in the alliance, and the promotion of the evolution of the TSA system.

3.6. Internal Models. A valid internal model can infer the future state and environment of agents to generate behavior and predict future results. The internal model is essentially a mechanism for the realization of predictions, and to some extent, it is equivalent to the schema proposed by Gell-Mann [71].

In a TSA system, when a large amount of information is input to an agent, that agent chooses and executes a certain mode to improve its adaptability. These modes are transformed into changes in internal structure in the process of implementation; that is to say, they form the ability to foresee and have a deep understanding of the future consequences of encountering similar modes. Specifically, in the governance process of a TSA, the alliance chooses different governance modes due to the influence of the degree of trust between partners [72]. Moreover, the organizational conventions within the system have some predictive power, but this is based on the accumulated experience of past events. Experience and convention are part of this internal model, but internal models of the TSA system require further exploration and research.

3.7. Building Blocks. Building blocks are the components of a CAS and its elements that have been reused in practice. They are constituted by basic agents in various ways, and they present their own characteristics [73]. Building blocks can be used to generate internal models. When a model is implicit, the process of discovering and combining building blocks develops through the evolution of the system. Different combinations of building blocks determine the adaptability of the system, and the mode of combination and the level of building blocks determine the complexity of the system.

A TSA is often composed of a range of building blocks, including enterprises, governments, universities, research institutions, and industry associations, each of which plays a 
different role. Enterprises have a dominant position in the TSA. They not only participate in technological development and are the owners of technology standards, but they are also the users of patents. The government provides funds and policy support to develop technology standards. Universities and research institutions are often the main participants in and providers of technological R\&D. Industry associations are intermediary organizations that provide service consultations and supervises TSAs. In a TSA system, agents at this level make up the next layer of building blocks. Taking enterprises as an example, the building blocks of enterprise hierarchy are core enterprises, participating enterprises, and peripheral enterprises [74], and their functions are different. Both core enterprises and participating enterprises are within the alliance and are distinguished according to the number of their patents and the status of their technology. Peripheral enterprises are users of standards. The next level of building blocks consists of different departments within each enterprise, and the next level after that involves individual employees. When a situation changes, the relevant building blocks are reassembled to adapt to it. For example, the deployment of employees is the arrangement of a combination of building blocks. In different combinations, the layered system produced in this way presents complexity.

\section{Echo Model for the TSA System}

As noted, a TSA can be seen as a CAS. The agent in a system has agentive initiatives and interacts with other agents to generate resources and make changes and adjustments to the environment. The echo model is a core model for CAS theory. It can explain the changes in the level and evolution of the TSA system as a result of interaction between agents and environment, which is especially suitable for the study of multiagent system evolution. We construct an echo model suitable for the TSA system to deeply analyze the phenomenon of emergence and the process of evolution of the system. We argue that a TSA is a contractual organization, with enterprises that set standards and pursue implementation, universities and scientific research institutions that provide auxiliary technology support, and government and industry associations that provide policy assistance. Because a TSA is enterprise-led, this paper focuses on characterizing the behavior of the enterprise agent on the basis of the echo model proposed by Holland and constructs an echo model suitable for a TSA system, including a basic echo model (model 1), an extended echo model (models $2-5$ ), and a three-layer echo model.

\subsection{Basic Echo Model}

4.1.1. Model 1: Attack Defense Mechanism. In the basic version of the echo model, attack and defense describe the mechanism of acquiring resources. The agent has two parts: a repository for resources and a chromosome string that represents behavioral competence. Each agent has only one chromosome, and the attack tag and defense tag are carved on it. The interactive activities of the agents in the model are regulated by these tags. Both the attack tag and the defense tag represent resources; specifically, the former represents the resources needed by the agent and the latter represents the resources owned by the agent. In relation to enterprise development technology standards, resources can be divided into five categories: $R_{1}$, human resources; $R_{2}$, financial resources; $R_{3}$, knowledge resources; $R_{4}$, information resources; and $R_{5}$ equipment resources. $R_{i j}$ represents specific resources, in the form $R_{11}, R_{12}$, etc.. When two agents meet, one attacks the other, matching its own attack tag to the other's defense tag. If the two tags have a high degree of match, they the agent can acquire most or all of the other's resources. Otherwise, it can only take the other's excess resources or nothing at all. In the model, we assume that the resources on the attack tag and defense tags either match each other plus 2 points, do not match, or exceed minus 1 point (based on the resources of the attack tag). The attack tag of each agent is marked with $N$ resource items, which are matched with the defense resources of other agents. If $n$ items of required resources can be provided to the agent, its matching score is $2 n-(N-n)=3 n-N$. For example,

The attack tag of agent 1 is $\left\{R_{11}, R_{12}, R_{21}, R_{22}, R_{23}, R_{41}\right\}$ The defense tag of agent 2 is $\left\{R_{11}, R_{22}, R_{23}, R_{41}, R_{51}\right\}$

The attack tag of agent 2 is $\left\{R_{12}, R_{13}, R_{21}, R_{33}\right\}$

The defense tag of agent 1 is $\left\{R_{13}, R_{14}, R_{31}, R_{32}, R_{33}, R_{42}\right\}$

When agent 1 and agent 2 meet, the attack tag of agent 1 is matched with the resources on the defense tag of agent 2. Agent 1 needs six kinds of resources, and agent 2 has five kinds of resources. Agent 2 can meet four of the kinds of resources needed by agent 1 . The matching score is $3 \times 4-6=6$ points. Similarly, the attack tag of agent 2 is matched to the defense tag of agent 1 , and the score is $3 \times 2-4=2$ points. Obviously, the degree of matching between agents is not equal. This degree determines the result of the resource, whether transfer, preservation, or surplus. Each agent is matched with agents that can be contacted, and then the agent with the highest matching score is selected for resource exchange. This basic model in the TSA system cannot fully present how agents decide whether to exchange resources or produce other complex phenomena in the evolution of this system. Thus, the echo model must be further expanded.

\subsection{Extended Echo Model}

4.2.1. Model 2: Resource Exchange Mechanism. In the resource exchange model, exchange conditions are added for the determination of whether to exchange resources, based on the matching degree of the agents. This model still follows the functions of chromosomes mentioned before, and it further refines the basic model, dividing chromosomes into tag and control segments. A tag segment matches the description above, including attack tag and defense tag. The new exchange conditions are found in the control segment. The formulation of technology standards is not done independently by one agent, and it requires constant interaction with other agents to achieve its output of technology 
standards. In the TSA system, agents do not unconditionally provide resources for other agents, and each exchanges its own surplus resources. When two agents in the model meet, the exchange conditions of each agent are checked in addition to consider the match with the attack tag of the other agent. Only when both conditions are met do the agents begin interactive activities for resource exchange. If one party's conditions are not met, it has the opportunity to escape interactive activities, which involves the probability of suspending them. That is, the agent is not always able to exchange resources with the most highly matched agent. In addition to the matching the attack and defense tags and the exchange conditions, resource exchange activities are also affected by the market environment.

\subsubsection{Model 3: Resource Conversion Mechanism. Resource} transformation mechanisms add a transformation subsegment to the control segment of the chromosome. This segment has an enzyme-like function, which converts a certain amount of resources into another specific resource. Essentially, companies adopt a series of actions to enhance their competitiveness and obtain more benefits. To share technological achievements and reduce the cost and risk of standardization [75], enterprises connect with partners such as enterprises, governments, universities and scientific research institutions, industry associations, and others to form a TSA. All enterprises in a TSA share the motivation of resource transformation, the purpose of which is to transform knowledge, information, talents, and other resources into technology standards and products and promote marketization and industrialization.

4.2.3. Model 4: Conditional Adhesion Mechanism. The cohesion model effectively explains the aggregation and evolution of the TSA system. To allow the echo model to achieve conditional adhesion, a new adhesion tag is added to the chromosome. When two agents meet, the adhesion tag of the one agent is matched to the attack tag of the other. If the matching score of the two agents is close to 0 , no adhesion occurs. However, so long as there is an agent matching score that is not 0 , adhesion will occur. Put simply, an agent conditionally chooses other agents to realize its connection, and it forms an aggregate with boundaries. Once this aggregate is formed, interactive activities become more complicated. In essence, business entities do not choose to connect with other companies with the same resources and technology capabilities, to better achieve adhesion. Agents tend to choose complementary agents to conduct connection cooperation, and they are, respectively, responsible for the development of different technology standards, to share risks and benefits. Within the TSA system, each agent achieves connection and adhesion with at least one agent, and they cooperate with each other to jointly develop and formulate technology standards. In adhesion, the level of the system is also spontaneously generated. When the agents stick to each other over a long period, a hierarchy emerges, that is to say, agents adopt full-time activity, and the agents in different hierarchies have special responsibilities to improve the efficiency of the development of standards.

4.2.4. Model 5: Agent Evolution Mechanism. The evolution of the TSA system is a dynamic process, and its internal agents are also undergoing constant evolution. The agent not only interacts with individual or multiple agents but also reacts to the external environment. Within the TSA system, each agent is located in an appropriate niche, defined by interactions centered on the agent. However, agents are evolving, and the role provided to other agents and alliance system is not fixed. After they enter the alliance, the agent gains the technology and other support needed for development. Through continuous learning, the agent changes its position in the alliance, and its role in technology standard setting activities changes with improvements or declines in capability and technology. The agent may move from one niche to another or from one system level to another, and it may even withdraw from the system.

The evolution of the agent in a TSA can be divided into three stages, as follows. First one is the agent entry. Suppose the probability of the agent entering the TSA during $t$ is $P_{\text {at }}$ and the critical probability that the system allows it to enter at this time is $P_{1}$. Then, when $P_{\text {at }} \geq P_{1}$, new agents enter the alliance. Second is the agent migration or the change of an agent from one level in the system to another. In the TSA system, all activities support formulating and spreading technological standards. The agent's role in the formulation of these standards or changes in the ability of these standards can cause the agent's position to shift. For example, for an agent of a government or industry association, when a standard changes or there is a major change in the external environment, its role changes, and so does its ecological niche within the alliance system. Third is the agent exit. The main purpose of joining the TSA is to enhance the agent's own technological capabilities, launch more products, and achieve marketization. If the agent fails to achieve its goals or fails to obtain benefits, it withdraws from the TSA.

4.3. Three-Layer Echo Model. The basic and extended models incorporate a detailed explanation of the interaction between the agent and the mechanisms of the aggregation of multiple agents to form the TSA. The agent forms a TSA by adhering to other agents. After forming the alliance system, the interaction between agents and the environment continues. This is an integrated effect of the different mechanisms within the system that promotes the evolution of the TSA. However, models 1 to 5 are largely restricted to explaining the local mechanisms of agent interaction within a TSA system. To better describe the overall evolution of this system, a three-layer echo model is constructed, by combining the matching degree mentioned several times in the previous model mechanism. The matching of tags is key to the echo model, and it is the prerequisite for interaction and cooperation in formulating technology standards. The relevant literature indicates that the degree of matching is affected by matching time, matching speed, and matching ability [76]. Based on these three dimensions, a three-layer 
echo model of the evolution of the TSA system is constructed, as shown in Figure 1.

4.3.1. Upper Layer: Matching Ability. In the interaction between agents, matching ability relates to both the acquisition and exchange of resources and to the transformation of resources and to agent cooperation. The stronger the agent's resource-matching ability is, the more cooperative agents can be selected and the wider the scope is of available resources, producing greater benefit to the development of standard setting. This entails that the upper-layer model of the evolution of the TSA, matching capability, is established here.

4.3.2. Middle Layer: Matching Speed. A middle-layer model is established that corresponds to the ability-matching speed. The main motivation for joining the TSA to carry out R\&D activities for a technology standard is ultimately to create greater benefit. In addition, the products are quickly updated, and it is only by developing new products more quickly that can they develop better returns. Therefore, the agent's resource-matching speed is very important. The quicker the matching speed of the agent's resources; the quicker its response ability; the faster its speed of resource acquisition, exchange, and transformation, the quicker its output of technology standards and products by the TSA.

4.3.3. Lower Layer: Matching Time. Obviously, a TSA system constantly evolves over time. An enterprise gradually evolves from a single agent at the beginning to form a TSA. The environment, agent, and alliance system are all dynamic, and their impacts at different points in time are not consistent. The agent's matching ability and matching speed also change over time, so matching time should be taken into account to establish the lower-layer model of the evolution of the TSA system.

In the three-layer echo model, the matching ability and matching speed of the agents may change with change in time, and change in matching speed may also cause a change in matching ability. Therefore, the upper-layer model is the result of the combined action of the middle-layer model and the lower-layer model.

In addition, we add the indicator alliance competency to describe the adaptability of the agent after its participation in the TSA. After joining the alliance, the agent gains a certain alliance competency, that is, an initial competency. For resource exchange, the connection and matching of the agent with other agents and the development of resource exchanges all have behavioral costs, that is, the agent loses a certain degree of competency in these transactions, namely, competency metabolism.

For the resource conversion mechanism, the agent converts the resources into technology standards and products and gains the benefits. The prerequisite for resource transformation is obtaining necessary resources for the development of technology standards. We use resource competency to express benefits. If the agent's resource competency is $X$, then its revenue increases by $X$ for each unit of resource added to the resource conversion.

In the conditional adhesion mechanism, the agent achieves adhesion with other agents when certain conditions are met, while the agent remains in continuous evolution. After joining the alliance, the agent may rapidly develop and realize an expansion of scale by constantly connecting and cooperating with other agents and frequently interacting with resources. We use promotion cost to represent the cost (competency) to the agent for each promotion:

$$
\begin{aligned}
\text { alliance competency }= & \text { initial competency } \\
& + \text { resource increment } \\
& \times \text { resource competency value } \\
& - \text { competency metabolism } \\
& - \text { promotion cost. }
\end{aligned}
$$

The agent's alliance competence indicates the evolutionary state of the agent. If alliance competence $>$ initial competence, the agent is well developed and can use the advantages of the TSA to continuously expand its own scale; if alliance competence < initial competence, the agent can cope with the competitive pressure of the market and maintain its own survival. However, when the agent's alliance competence decreases to 0 , this means that the agent has withdrawn from the TSA. In other words, alliance competence reflects the agent's ability to adapt to other agents in the TSA and the external environment. The core of this model is the expression of the agent's resource acquisition, exchange, transformation, conditional adhesion, and evolution as its adaptability to provide a solution for studying how the agent's adaptive behavior evolves into a complex TSA system.

\section{Simulation Analysis}

The above echo model describes the evolutionary mechanism of a TSA system from the perspective of the interaction behavior of agents. This paper uses agent-based modeling and simulation [77] to study the complex system and simulates the evolution of the TSA system through NetLogo programming. NetLogo $[78,79]$ is a programming language and modeling platform used to simulate natural and social phenomena, proposed by Uri Wilensky in 1999 and developed by the Center for Connected Learning and Computer-Based Modeling of Northwestern University. It is especially suitable for simulating complex systems that develop over time. The introduction of random factors into the simulation gives its results improved ability to describe and express what it is simulating [80].

In this paper, the echo model of TSA system evolution is programmed and simulated in NetLogo 6.1.1, the latest version of NetLogo. The agents in the simulation model are cows, patches, and observers. Cows are operators (agents), which can be used to simulate humans, enterprises, or anything else, and they move within patches. Patches, for their part, make up a grid environment and are an activity space for the agents and can be used to signify grassland, 


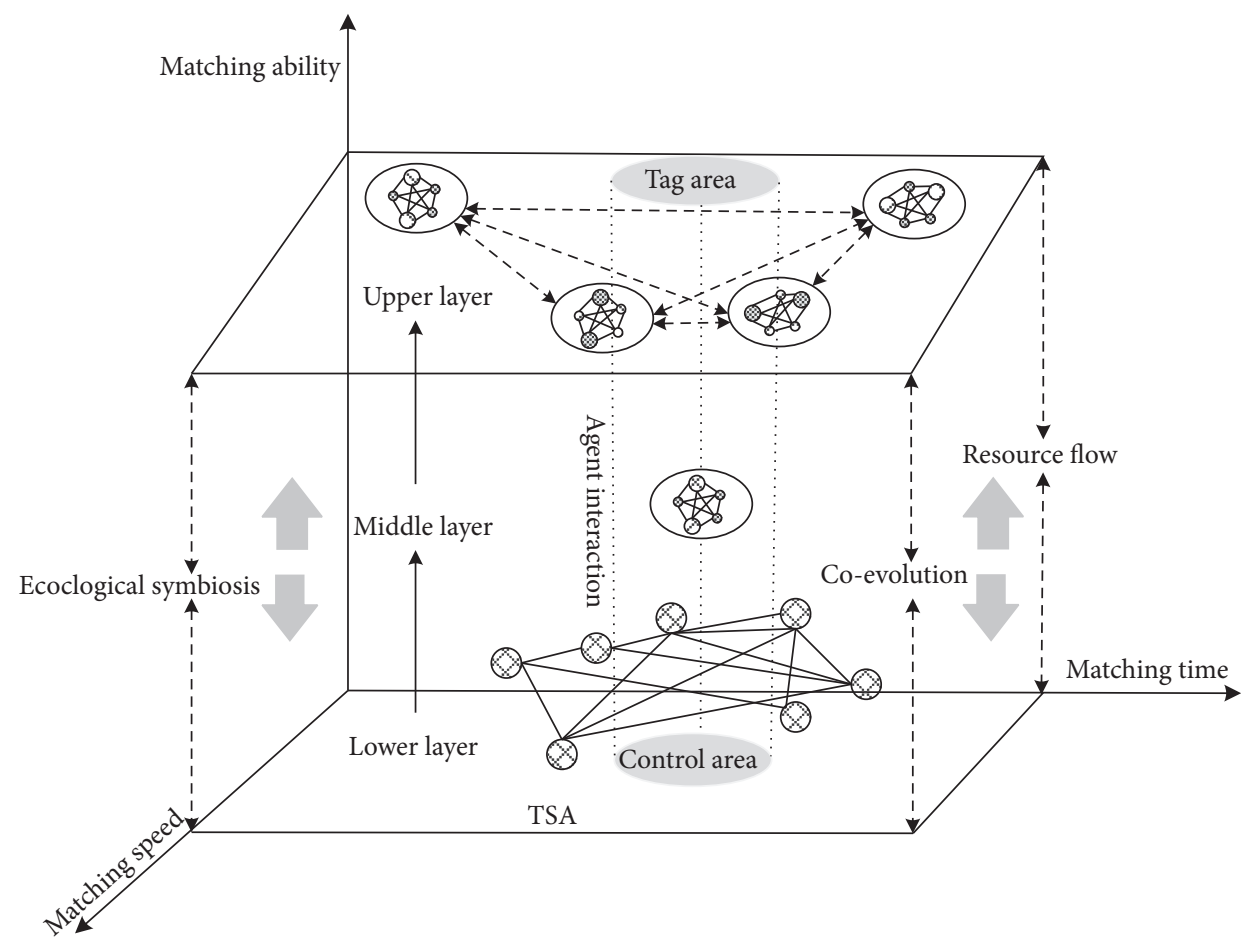

Figure 1: Three-layer echo model.

resources, or anything else. Observers are the issuers and coordinators of the main commands and the observers of the state of the simulation. In the simulation model of TSA, enterprises are the cows, and universities, scientific research institutions, government, and industry associations are part of the environment. In the TSA system, interactions and influences between agents and the environment are the main driving force of the system's evolution. Therefore, to achieve a simulation that can model the evolution process of TSA system, using NetLogo software, we set rules for behavior between one agent and another, between an agent and its environment, and among environmental variables, to make the agents evolve complex behavioral phenomena under the environment and set rules.

Based on the echo model and simulation conditions, the evolution process of the TSA system is simulated. The entire simulation experiment focuses on the angle of the agents' adhesion aggregation and resource interaction to simulate the evolution process of the TSA system.

5.1. Agent Aggregation Evolution Simulation. A prominent feature of the TSA seen as a CAS is the formation of a complex hierarchical structure among its agents. We simulate the connection hierarchy of agents in the process of forming TSA aggregation. The agents achieve long-term connections through the adhesive mechanism and thus form the TSA. The simulation model mainly incorporates the rules of the agents and simply presents an aggregate evolutionary process of the TSA system from a macroscopic view. It is assumed that all agents in the space maybe join the TSA. Due to the limitations of the simulation interface, the model only shows adhesion and aggregation of a few agents. To facilitate the simulation of adhesion, the agents are arranged randomly in a circle, and their adhesion is carried out in the middle position. Gray and red components represent agents (here, we only consider the relevant agents that may aggregate to form a TSA in the industrial environment). A gray line indicates where agents reach a short-term connection, and a red line means that a long-term connection between agents has been achieved, that is, adhesion has been achieved. Moreover, we call the number of agents directly connected to an agent the degree of that agent.

TSAs are usually initiated by enterprises with strong technological ability and a high market position. In the initial simulation state, as shown in Figure 2, the simulation interface shows the distribution of agent adhesion when the TSA is launched. We can see that 11 red agents have reached adhesion and are ready to unite with more agents to form a TSA. Two agents have a degree of 3 , five agents have a degree of 2 , and the degree of the remaining four is 1 .

As the simulation experiment develops, more and more agents join in, and the aggregate expands. The intermediate aggregate is shown in Figure 3. At this point, the adhesion of agents in the aggregation is different, and a more complex network connection structure is formed. Moreover, in the dynamic aggregation of agents to form the TSA, it can be observed that a new aggregation may not be formed around a single agent but may be a multiagent that achieves a shortterm connection. Then, the aggregation continues to evolve, and agents aggregate and form a TSA, as shown in Figure 4. We can see that other agents appear outside the TSA system. At this time, the evolution of the TSA system has not stopped, and agents continue to enter and exit.

The degree of distribution of the aggregate also indirectly indicates the aggregate connection among agents. In the 


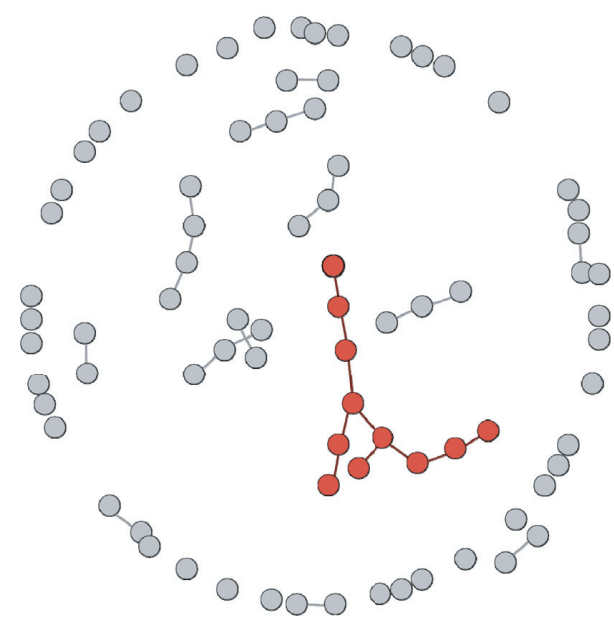

Figure 2: Initial state of alliance initiation.

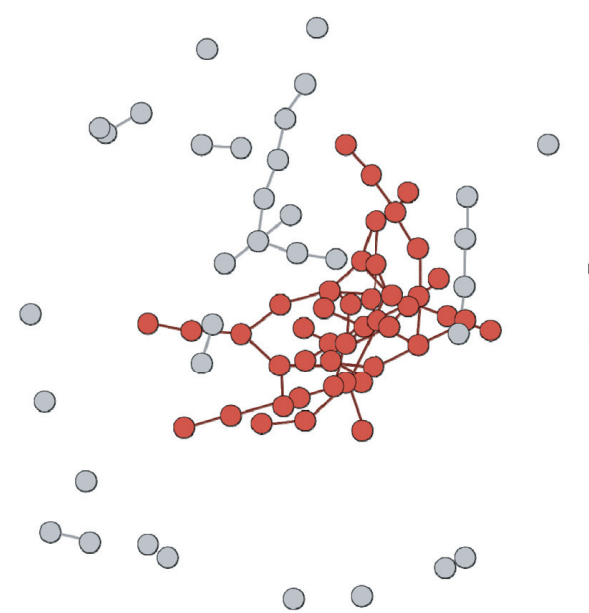

FIgURE 3: Intermediate state of alliance development.

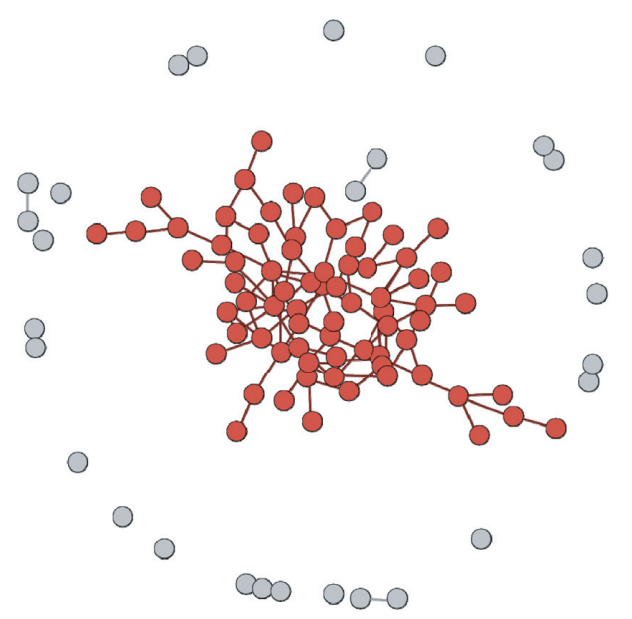

Figure 4: Stable state of alliance development.

simulation experiment, it is seen that, when the number of agents is 20 , the distribution of degree of a time node aggregate is as given in Figure 5. In the aggregate network,

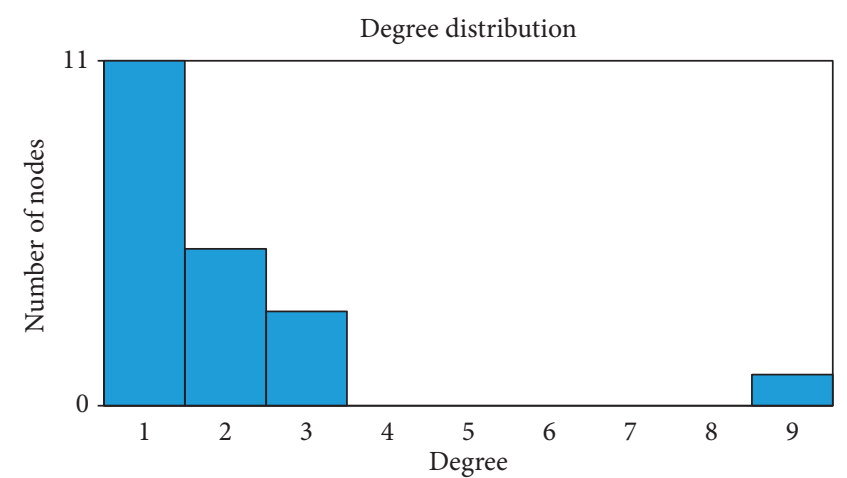

FIgURE 5: Degree distribution of the aggregate when the number of agents is 20 .

there are 11 agents that only adhering to a single agent, and the maximum degree for an agent is 9. After the adhesion of agents, the number of agents in the aggregate continues to increase, and when a certain critical condition is reached, the TSA evolves and forms. Over the entire simulation, the number of agents with an aggregation degree of 1 is the largest and the connection between agents changes over time. These results show that the hierarchical structure of a TSA system is dynamic and changes with adhesion between the agents.

5.2. Agent Interaction Evolution Simulation. To better highlight the role of the basic model of agent interaction, namely, the attack defense mechanism, we divide enterprises in the industrial environment into two categories: cooperative and greedy agents. Cooperative agents are those that have joined a TSA and develop in coordination with the alliance as a whole. Greedy agents are those that are outside the alliance and pursue their own development, only connecting with cooperative agents for a short time. In the initial state, a small number of agents with core technologies become connected to initiate a TSA. At this time, the relationship between agents is not stable. In the simulation model, each agent is randomly assigned an initial position, and cooperative agents (in orange) and greedy agents (in blue) are distinguished by the color of the cows. Each patch represents the resources of one unit and is located in a different ecological niche. The main parameter settings and value ranges are presented in Table 1.

The evolution of a TSA system is inseparable from the interactions between the agents and the environment, that is, the flow of resources. In the simulation model, we set competency values for the agents to measure the survivability of each in the TSA system, and we took a competency value of 0 as the critical condition for the agent to exit the system. The simulation interface included the TSA system and its external environment. There were 13 cooperative agents and 7 greedy agents in the system, and the parameters of each agent were retained at the initial value. The change in the number of agents at the early stage of the simulation operation is given in Figure 6. At time steps $0-14$, the number of agents in both categories is in the stage of rapid growth, and the growth rate 
TABLE 1: Simulation parameter setting.

\begin{tabular}{|c|c|c|c|}
\hline Parameter & Parameter description & $\begin{array}{l}\text { Initial } \\
\text { value }\end{array}$ & $\begin{array}{l}\text { Value } \\
\text { range }\end{array}$ \\
\hline Cooperative agent & The agent in a TSA & 13 & - \\
\hline Greedy agent & The agent connected with the agents in the alliance for a short time & 7 & - \\
\hline Matching ability & Determines the flow range of the agent in a time step & 0.02 & $0 \sim 0.3$ \\
\hline Resource competency & Equivalent to behavior income & 50 & $0 \sim 200$ \\
\hline $\begin{array}{l}\text { Competency } \\
\text { metabolism }\end{array}$ & Equivalent to behavior cost & 6 & $0 \sim 99$ \\
\hline Promotion cost & Each agent needs to pay a certain competency cost for each promotion & 60 & $0 \sim 99$ \\
\hline Promotion threshold & The amount of basic resources an agent must have in order to be promoted & 100 & $0 \sim 200$ \\
\hline Low-high-threshold & Resource regeneration threshold & 5 & $0 \sim 99$ \\
\hline Low-growth-chance & $\begin{array}{c}\text { Above low-high-threshold, the renewable resource is calculated with high-growth- } \\
\text { chance }\end{array}$ & 30 & $0 \sim 99$ \\
\hline High-growth-chance & Below low-high-threshold, resource regeneration is calculated as low-growth-chance & 70 & $0 \sim 99$ \\
\hline Max-resource-height & Maximum number of resources & 10 & $0 \sim 40$ \\
\hline Cooperative probability & Setting an appropriate probability of cooperation between the agents & 0.6 & $0 \sim 1.0$ \\
\hline
\end{tabular}

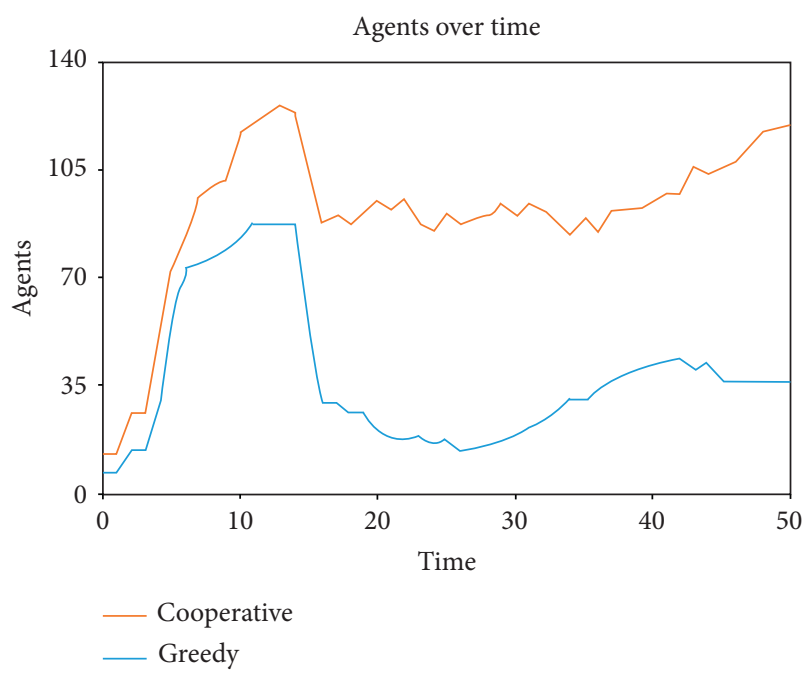

FIGURE 6: Early simulation interface.

is basically the same. This indicates that the number of agents is small in the initial stages of the evolution of a TSA system. On the basis of limited resources and R\&D ability, it is not sufficient for a few agents to develop technology standards alone. Therefore, agents in the aggregation body need to seek partners including cooperative agents willing to join the TSA as well as greedy agents that are only seeking a short-term connection, after which the aggregation scale rapidly expands. At time step 14, the inflection point occurs for the quantity of change for both types of agents. In the early stages of the evolution of a TSA system, some agents' competency decreases to 0 after a series of interactive activities, and these withdraw from the system. As the number of entering agents falls below the number of exiting agents, the number of both types of agents decreases. After a short decrease, the number of cooperative agents stabilized at about 90 and began to rise in volatility at time step 44, indicating an overall increase in cooperative agents. After time step 14, the fluctuation of greedy agents decreases, and in the later stages of the simulation, the number of greedy agents decreases to 0 , as shown in Figure 7. Finally, the agents in the TSA can meet their own

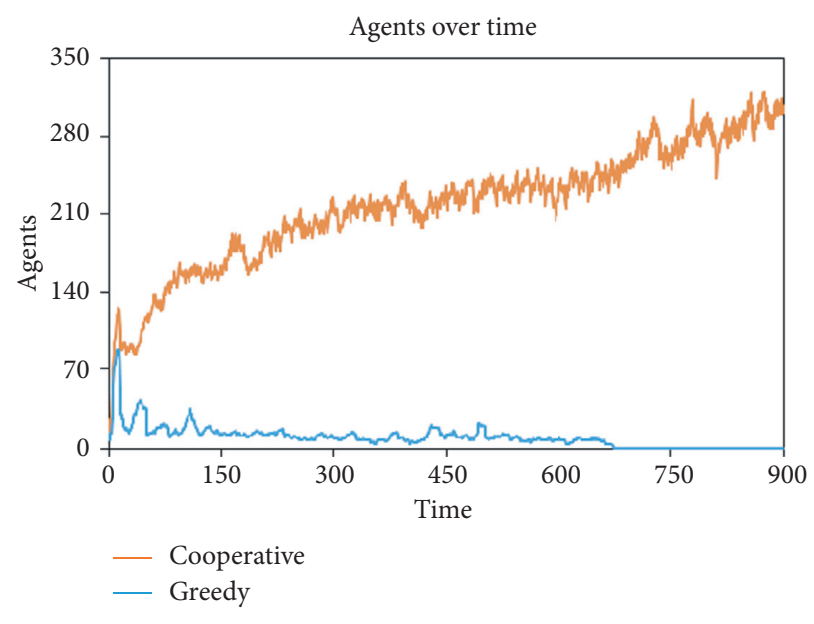

Figure 7: Later simulation interface.

resource needs in resource interactions with other agents or the system environment, and they have no further need to find short-term connections with external greedy agents to obtain resources. From this point, the agents in the TSA are committed to jointly developing, formulating, and diffusing technology standards, such that the TSA has reached a relatively stable state. In the following time step, agents interact constantly, and the TSA still accommodates the entry and exit of agents.

In many simulation experiments, the distribution of agents across the simulation interface is unequal. The setting of the matching ability of agents and the limitations of the scope of matching resources alter the simulation results. However, the following simulation phenomena are not affected.

With matching ability as the independent variable, other variables can be controlled to remain unchanged in the initial state. We observe the evolution state of TSA by adjusting the value of the matching ability. Figure 8 clearly shows that, when the matching ability is 0.01 , the number of agents changes with the time step. When the time step is 50, the number of cooperative agents in the TSA is 103 and the 


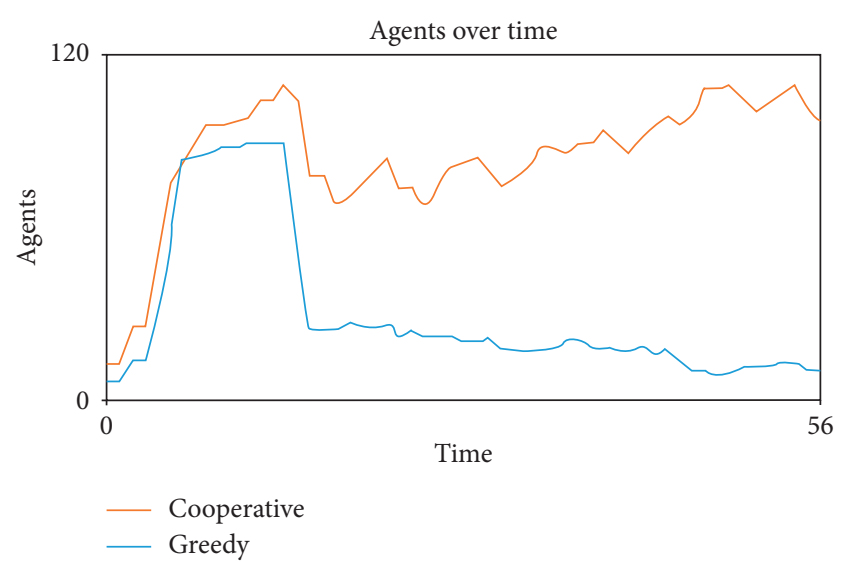

FIGURE 8: Matching ability $=0.01$.

number of greedy agents is 11 . In Figure 9, the evolution of the entry and exit of agents of the TSA is shown when the matching ability is 0.03 . At time step 50, the number of cooperative agents is 178 and the number of greedy agents is 31. It is clear that the evolution scale of the TSA is also larger, due to the increased resource-matching ability among the agents. We believe that the stronger an agent's resourcematching ability is, the faster is its rate of resource acquisition, exchange, and transformation. To maintain stronger resource-matching capability, at each time step, an agent requires additional resources and needs to adhere to more other agents to meet the needs of its own technology development. Therefore, the stronger the resource-matching ability of the internal agents and the larger the number of cooperative agents adhering to the aggregation, the larger the development scale of the TSA. However, the larger the scale of the alliance, the more the agent needs to improve its adaptability to be able to match resources with more agents.

Resource competency is taken as the independent variable, and the other variables are controlled in the initial state and remain unchanged. For a single agent, the higher the resource competency, the better the promotion. Higher competency of the agent's resources entails that the agent produces more results for a unit of resources, that is, having a higher conversion rate of resources, which is more conducive to the development of the agent. However, this is not the case for the TSA system as a whole. In the simulation experiment, when resource competency is 50 , the change trend of the number of agents is smaller, and the number of agents at the same time is greater than at other levels of resource competency. At time step 50, the number of cooperative agents is 130 when the resource competence is 50 . When the resource competency is 40 or 60 , the number of cooperative agents is 95 or 106, respectively. We believe that when resource competency is low, the development of the agents and alliance system is limited and evolution is slower. However, when resource competence is too high, the agent conflict is more obvious and the change trend of the number of agents is steeper. This indicates that agents enter and exit more frequently over the same period of time, and the agents in the alliance always seek to change their own adaptability to adapt to new agents, which is not conducive to the stable

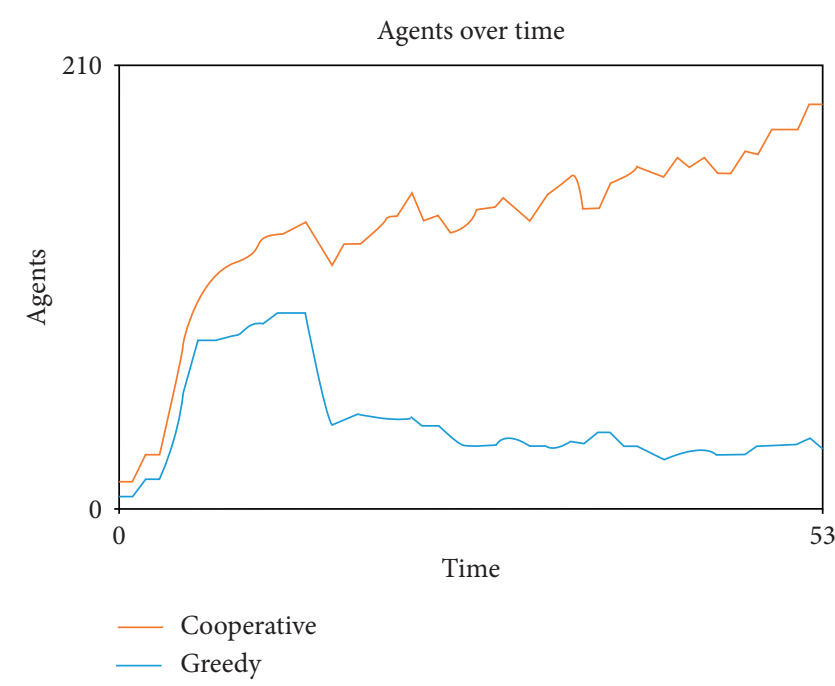

Figure 9: Matching ability $=0.03$.

development of the TSA. However, if the resource competence of the agents is moderate, the scale of the alliance is larger, and it becomes more stable, which is beneficial to its overall development.

Competency metabolism is taken as the independent variable, and other variables are controlled in the initial state and remain unchanged. The lower the competency metabolism is, the lower the flow cost of the agent in the simulation model, that is to say, the competency cost paid by the agent to obtain the same amount of resources is less. We set the competency metabolism to different values, that is, to 4 , $5,6,7$, and 8 , and through simulation it is observed that when the system reaches step 50, the number of cooperative agents is $189,123,110,101$, and 85 , respectively. It can be concluded that when competency metabolism is lower, with the growth in time, the more agents in the TSA system, the larger the alliance scale is after development stabilizes.

\section{Conclusion}

6.1. Research Conclusions. In this paper, CAS theory is used to study the evolution of the TSA system. Essentially, a TSA can be understood as a kind of CAS by analyzing its complex adaptive characteristics. An echo model is reconstructed according to the evolution of a TSA, and the interaction mechanisms of the agents and the emergence of the system hierarchy are explained. The evolution process is reproduced in a NetLogo programming simulation, and the characteristics of the CAS are verified and explained. The key factors affecting the evolution of TSA are extracted. The conclusions of this study are as follows:

First, a TSA is a dynamic, complex, and multiagent CAS, which includes enterprises, governments, universities, and scientific research institutions. Whether it is in the early stages of a TSA or in its later stages of stable development, agents are always entering and exiting. The interaction between the agents within the system and the environment makes the study of a TSA 
complex. However, each agent has subjective initiative. In response to the uncertainty of its external environment, an agent takes corresponding actions in standardization cooperation to adapt to changes in the environment.

Second, the simulation results of the echo model demonstrate that a complex hierarchical structure is formed among the agents in an alliance, which changes with changes in adhesion between the agents. With the evolution of a TSA, this is inseparable from the flow of resources between agents and the environment, and the change of the number of agents presents a nonlinear pattern of growth. In addition, under certain environmental conditions, the TSA evolves to a certain scale, with are full-link agents responsible for its development, formulation, and diffusion within the alliance, making it unnecessary to obtain resources from enterprises outside an alliance to complete the formation of technology standards.

Third, the resource-matching ability of each agent is a key factor in the evolution of a TSA. Matching capability means the ability to acquire, exchange, and transform resources. Low matching ability means that an enterprise agent has limited access to resources, making the resource transformation activities limited, and the development of technology standards will progress slowly. This affects the evolution of the TSA. With high matching ability, moderate behavior income and low behavior cost, an agent can carry out resource interaction with more agents and adhere to more agents, thus promoting the evolution process of its TSA.

6.2. Discussions and Prospects. The above conclusions have certain significance for guiding the development and evolution of TSA. Essentially, our findings indicate that a TSA is a typical CAS, with a complex hierarchical structure. Moreover, enterprise agents continuously interact with other agents and the environment (through resource matching), which promotes the dynamic evolution and development of the TSA. Our findings support certain conclusions from previous studies. Consistent with the views of Narayanan and Chen [81], we argue that facilitating standards in complex technological systems requires the collective effort of various agents. Likewise, standardization alliances evolve through collaboration among firms to develop and implement industry technical standards [82]. In line with the conclusion of Rodon et al. [83], we further propose that it is only when the resources of enterprises in the TSA are dynamically matched with the resources required at different stages of the process can any standardization be smoothly implemented. Moreover, this paper develops the description of the evolution mechanism of the whole system on the basis of previous studies [84-86]. Additionally, changes in the external environment are also taken into account among the influencing factors of the TSA system.

Applying CAS theory, this paper not only describes the interaction between agents but also clearly presents the interaction between agents and the environment, studying the influence of internal and external factors on the evolution of TSA. It also untangles the reason why resource integration is the main influencing factor in alliance evolution and development [87]. The interaction between the alliance and the environment is accomplished through the interaction between the agents and the environment. The continuous acquisition, exchange, and integration of the main resources are the driving force of the evolution and development of the TSA. Further, our results indicate that the interaction between agents and the environment is very important. Enterprises should optimize their resource structure and improve their adaptability to the environment to reach better development in a TSA.

Besides, we admit that this paper also has some limitations. First, it focuses on the macro level, with less consideration being given to the subjective factors in the relationship between alliance agents. In the future, we intend to divide the evolutionary stages of the alliance agent more carefully in combination with relationship quality and agreement mode between alliance partners to improve study of the evolution and development of the TSA. Second, in the part of echo model construction, we present a measurement method for alliance competency (that is, the adaptability of an agent to the alliance), rather than providing the mathematical model of a multiagent. The TSA system, which can be regarded as an ecosystem, includes not only the internal adaptive agents but also the external environment. Combined with the simulation method, this can clearly indicate the specific impacts of resource interaction between agents and environment in a TSA system. This paper aims to provide a reference direction for the study of strategic alliances from a system perspective. In the future, we intend to use mathematical models to test our understanding of the evolution of the agents and the alliance system and to verify the reliability of our conclusions with empirical methods. In addition, in the simulation of the TSA system evolution, we only studied the evolution of adaptive agent aggregation and interactive evolution processes. A parameter index setting may be lacking. In the future, we may review other simulation platform software to present the entire process of TSA evolution more comprehensively.

\section{Data Availability}

The data used to support the findings of this study are available from the corresponding author upon request.

\section{Conflicts of Interest}

The authors declare that they have no conflicts of interest.

\section{Acknowledgments}

This research was supported by the National Natural Science Foundation of China (71774067).

\section{References}

[1] J. Wonglimpiyarat, "Standard competition: is collaborative strategy necessary in shaping the smart card market?" 
Technological Forecasting and Social Change, vol. 72, no. 8, pp. 1001-1010, 2005.

[2] H. Jiang, S. Gao, S. Zhao, and H. Chen, "Competition of technology standards in industry 4.0: an innovation ecosystem perspective," Systems Research And Behavioral Science, vol. 37, no. 4, pp. 772-783, 2020.

[3] G. Tassey, "Standardization in technology-based markets," Research Policy, vol. 29, no. 4-5, pp. 587-602, 2000.

[4] S. K. Zhao, H. Q. Yu, and H. Jiang, "Study on the relationship between technology standard, technological innovation and economic growth-theoretical model and empirical analysis," Studies in Science of Science, vol. 30, no. 9, pp. 1333-1341, 2012.

[5] D. P. Wang, X. Y. Wei, and Z. D. Zhang, "Study on value evaluation of technology standard based on innovation ecosystem of hi-tech enterprises," China Soft Science, vol. 11, pp. 40-48, 2013.

[6] P. Kale, H. Singh, and H. Perlmutter, "Learning and protection of proprietary assets in strategic alliances: building relational capital," Strategic Management Journal, vol. 21, no. 3, pp. 217-237, 2000.

[7] Y. Su and T. C. Li, "Simulation analysis of knowledge transfer in a knowledge alliance based on a circular surface radiator model," Complexity, vol. 2020, Article ID 4301489, 27 pages, 2020.

[8] R. Axelrod, W. Mitchell, R. E. Thomas, D. S. Bennett, and E. Bruderer, "Coalition formation in standard-setting alliances," Management Science, vol. 41, no. 9, pp. 1493-1508, 1995.

[9] G. Andrevski, D. J. Brass, and W. J. Ferrier, "Alliance portfolio configurations and competitive action frequency," Journal of Management, vol. 42, no. 4, pp. 811-837, 2013.

[10] C. C. Phelps, "A longitudinal study of the influence of alliance network structure and composition on firm exploratory innovation," Academy of Management Journal, vol. 53, no. 4, pp. 890-913, 2010.

[11] T. A. Hemphill, Cooperative Strategy and Technology Standards-Setting: A Study of United States Wireless Telecommunications Industry Standards Development, The George Washington University, Washington, DC, USA, 2005.

[12] A. C. Inkpen, "Learning and knowledge acquisition through international strategic alliances," Academy of Management Perspectives, vol. 12, no. 4, pp. 69-80, 1998.

[13] B. Antoncic and I. Prodan, "Alliances, corporate technological entrepreneurship and firm performance: testing a model on manufacturing firms," Technovation, vol. 28, no. 5, pp. 257-265, 2008.

[14] R. M. Monczka, R. J. Trent, and R. B. Handfield, Purchasingand Supply Chain Management, South-western College Publishing, Cincinnati, OH, USA, 1998.

[15] T. K. Das and B. S. Teng, "The dynamics of alliance conditions in the alliance development process," Journal of Management Studies, vol. 39, no. 5, pp. 728-746, 2002.

[16] M. Sarkar, R. Echambadi, S. T. Cavusgil, and P. S. Aulakh, "The influence of complementarity, compatibility, and relationship capital on alliance performance," Journal of the Academy of Marketing Science, vol. 29, no. 4, pp. 358-373, 2001.

[17] T. Keil, "De-facto standardization through alliances-lessons from bluetooth," Telecommunications Policy, vol. 26, no. 3-4, pp. 205-213, 2002.

[18] T. K. Das and B.-S. Teng, "Between trust and control: developing confidence in partner cooperation in alliances," The
Academy of Management Review, vol. 23, no. 3, pp. 491-512, 1998.

[19] H. Jiang, S. Y. Sun, and Y. H. Wu, "Research on the evolution mechanism of knowledge ecosystem in technical standards alliance-case of IGRS industry alliance," Science \& Technology Progress and Policy, vol. 36, no. 21, pp. 1-9, 2019.

[20] B. C. Liu, "Research on collaborative innovation evolution process and innovation mechanism framework construction of strategic emerging industry technology standards alliance," Knowledge Economy, vol. 10, pp. 23-24, 2019.

[21] T. A. Hemphill, "Technology standards-setting in the US wireless telecommunications industry: a study of three generations of digital standards development," Telematics and Informatics, vol. 26, no. 1, pp. 103-124, 2009.

[22] F. F. Suarez, "Battles for technological dominance: an integrative framework," Research Policy, vol. 33, no. 2, pp. 271-286, 2004.

[23] A. E. Akgün, H. Keskin, J. C. Byrne, and Ö. Ö. Ilhan, "Complex adaptive system mechanisms, adaptive management practices, and firm product innovativeness," R\&D Management, vol. 44, no. 1, pp. 18-41, 2014.

[24] S. A. Kauffman, The Origins of Order: Self-Organization and Selection in Evolution, Oxford University Press, New York, NY, USA, 1993.

[25] J. N. Warfield, "Twenty laws of complexity: science applicable in organization," Systems Research and Behavioral Science, vol. 16, no. 1, pp. 3-40, 1998.

[26] O. Babaoglu, H. Meling, and A. Montresor, “Anthill: a framework for the development of agent-based peer-to-peer systems," Distributed Computing Systems, vol. 7, pp. 15-22, 2002.

[27] J. Holland, Hidden Order: How Adaptation Builds Complexity, Shanghai Scientific \& Technological Education Publishing House, Shanghai, China, 1995.

[28] H. P. P. Hennie Lótter, "The complexity of science," Koers, vol. 64, no. 4, pp. 499-520, 1999.

[29] M. Mitchell Waldrop, "Complexity science," Red Herring Magazine, vol. 1, 2003.

[30] K. J. Laverty, "Managerial myopia or systemic short-termism?" Management Decision, vol. 42, no. 8, pp. 949-962, 2004.

[31] I. M. Cockburn, R. M. Henderson, and S. Stern, "Untangling the origins of competitive advantage," Strategic Management Journal, vol. 21, no. 10-11, pp. 1123-1145, 2000.

[32] Y. A. Zhang and C. G. Li, "Outlook for the researches of complex adaptive system's application domain," Management Review, vol. 22, no. 5, pp. 121-128, 2010.

[33] M. M. Waldrop, Complexity: The Emerging Science at the Edge of Order and Chaos, Simon and Schuster, New York, NY, USA, 1992.

[34] A. M. Wildberger, "Complex adaptive systems concepts and power industry applications," Control Systems Magazine, vol. 17, no. 6, pp. 77-88, 1997.

[35] H. J. Scholl, "Agent-based and system dynamics modeling: a call for cross study and joint research," in Proceedings of the 34th Hawaii International Conf on System Science, Maui, HI, USA, January 2001.

[36] J. H. Holland, Complex Adaptive System, Addison Wesley, Boston, MA, USA, 1995.

[37] D. R. Hofstadter, Gödel, Escher, Bach: An Eternal Golden Braid, Basic Books, New York, NY, USA, 1979.

[38] H. Jiang, W. T. Liu, and S. Y. Sun, "Knowledge integration capability, alliance management capability and technical standard alliance performance," Studies in Science of Science, vol. 37, no. 9, pp. 1617-1625, 2019. 
[39] L. F. Zhang, “The impact of technology standard alliance on the industry economic benefits-evidence from IGRS alliance and 3C industry," Forum on Science and Technology in China, vol. 12, pp. 87-95, 2018.

[40] T. H. Chiles, D. M. Alan, and J. H. Thomas, "Organizational emergence: the origin and transformation of Branson," Organization Science, vol. 12, no. 3, pp. 53-75, 2004.

[41] D. A. Levinthal, "Adaptation on rugged landscapes," Management Science, vol. 43, no. 7, pp. 934-950, 1997.

[42] K. Frenken, "A complexity approach to innovation networks. The case of the aircraft industry (1909-1997)," Research Policy, vol. 29, no. 2, pp. 257-272, 2003.

[43] L. Zhao and J. D. Aram, "Networking and growth of young technology-intensive ventures in China," Journal of Business Venturing, vol. 10, no. 5, pp. 349-370, 1995.

[44] D. Obstfeld, "Social networks, the tertius iungens orientation, and involvement in innovation," Administrative Science Quarterly, vol. 50, no. 1, pp. 100-130, 2005.

[45] B. Uzzi and J. Spiro, "Collaboration and creativity: the small world problem," The American Journal of Sociology, vol. 111, no. 2, pp. 447-504, 2005.

[46] N. Friedkin, "A test of structural features of Granovetter's strength of weak ties theory," Social Networks, vol. 2, no. 4, pp. 411-422, 1980.

[47] J. Hu, Y. Zhang, and X. Fang, "Research on partner selection mechanism of technological standard alliance: from the perspective of network embeddedness," in Proceedings of the Portland: Picmet'15 Portland International Center for Management of Engineering and Technology, pp. 585-595, Portland, OR, USA, August 2015.

[48] C. Takanashi and K. Lee, "Standard development by committees and communities: a comparative case study of IEEE1394 and USB," Technology Analysis and Strategic Management, vol. 25, no. 1, pp. 91-105, 2013.

[49] V. Chiesa and G. Toletti, "Standard-setting strategies in the multimedia sector," International Journal of Innovation Management, vol. 7, no. 3, pp. 281-308, 2003.

[50] B. Cees and Z. Fardad, "R\&D cooperation, partner diversity, and innovation performance: an empirical analysis," Journal of Product Innovation Management, vol. 31, no. 2, pp. 292312, 2014.

[51] R. Ram and R. Lori, "Do ties really bind? The effect of knowledge and commercialization networks on opposition to standards," Academy of Management Journal, vol. 57, no. 2, pp. 515-540, 2014.

[52] R. Cowan, N. Jonard, and M. Özman, "Knowledge dynamics in a network industry," Technological Forecasting and Social Change, vol. 71, no. 5, pp. 469-484, 2004.

[53] Y. Su and X. L. An, "Application of threshold regression analysis to study the impact of regional technological innovation level on sustainable development," Renewable \& Sustainable Energy Reviews, vol. 89, pp. 27-32, 2018.

[54] Y. W. Sun and Y. P. Wei, "Study on knowledge diffusion of high-tech enterprise alliance from the small-world network perspective," Journal of Management Sciences in China, vol. 14, no. 12, pp. 17-26, 2011.

[55] B. Wu and C. Z. Wang, "The influence of resource interdependence and relational quality on alliance performance: an empirical research," Studies in Science of Science, vol. 25, no. 2, pp. 334-339, 2007.

[56] K. Y. Shou, J. Wei, and Y. Liu, "Alliance portfolio diversity configuration for latecomers: qualitative comparative analysis," Studies in Science of Science, vol. 36, no. 7, pp. 1254-1263, 2018.
[57] W. Li, "Nature of standard-setting alliance: based on comparisons between R\&D alliance and patent alliance," Science Research Management, vol. 35, no. 10, pp. 49-56, 2014.

[58] O. Bruyaka and R. Durand, "Sell-off or shut-down? Alliance portfolio diversity and two types of high tech firms' exit," Strategic Organization, vol. 10, no. 1, pp. 7-30, 2012.

[59] T. D. Leeuw, B. Lokshin, and G. Duysters, "Returns to alliance portfolio diversity: the relative effects of partner diversity on firm's innovative performance and productivity," Journal of Business Research, vol. 67, no. 9, pp. 1839-1849, 2014.

[60] N. Lahiri and S. Narayanan, "Vertical integration, innovation, and alliance portfolio size: implications for firm performance," Strategic Management Journal, vol. 34, no. 9, pp. 1042-1064, 2013.

[61] J. Wei, Y. Ying, and Y. Liu, "R\&D geographic dispersion, technology diversity, and innovation performance," Studies in Science of Science, vol. 31, no. 5, pp. 772-779, 2013.

[62] G. Duysters and B. Lokshin, "Determinants of alliance portfolio complexity and its effect on innovative performance of companies," Journal of Product Innovation Management, vol. 28, no. 4, pp. 570-585, 2011.

[63] I. Castro, J. L. Roldán, and F. J. Acedo, "The dimensions of alliance portfolio configuration: a mediation model," Journal of Management \& Organization, vol. 21, no. 2, pp. 176-202, 2015.

[64] D. Lavie and S. R. Miller, "Alliance portfolio internationalization and firm performance," Organization Science, vol. 19, no. 4, pp. 623-646, 2008.

[65] G. Duysters, K. H. Heimeriks, B. Lokshin, E. Meijer, and A. Sabidussi, "Do firms learn to manage alliance portfolio diversity? The diversity-performance relationship and the moderating effects of experience and capability," European Management Review, vol. 9, no. 3, pp. 139-152, 2012.

[66] R. J. Jiang, Q. T. Tao, and M. D. Santoro, “Alliance portfolio diversity and firm performance," Strategic Management Journal, vol. 31, no. 10, pp. 1136-1144, 2010.

[67] J. Y. Wen and D. M. Zeng, "Alliance portfolio configuration and firm's technology standardization capability," Studies in Science of Science, vol. 37, no. 7, pp. 1277-1285, 2019.

[68] Y. Deng, "Market or relationship dependency? The effect of alliance partner selection orientation on technology innovation," Foreign Economics \& Management, vol. 38, no. 5, pp. 18-31, 2016.

[69] U. Wassmer and P. Dussauge, "Network resource stocks and flows: how do alliance portfolios affect the value of new alliance formations?" Strategic Management Journal, vol. 33, no. 7, pp. 871-883, 2012.

[70] T. K. Das and B.-S. Teng, "Partner analysis and alliance performance," Scandinavian Journal of Management, vol. 19, no. 3, pp. 279-308, 2003.

[71] M. Gell-Mann, The Quark and the Jaguar: Adventures in the Simple and the Complex, W. H. Freeman, New York, NY, USA, 1994.

[72] L. Xu, Y. Long, Z. Y. Zhang et al., "The impact of trust on the mode of alliance governance: a case study on China silian goup," Management Review, vol. 21, no. 7, pp. 121-128, 2009.

[73] P. Anderson, "Perspective: complexity theory and organization science," Organization Science, vol. 10, no. 3, pp. 216-232, 1999.

[74] Q. M. Li, J. P. Yang, and H. X. Zhao, "Research of how the competition of intra-cluster and inter-cluster, network externality of standard influence the willingness to form a standard alliance and its innovation performance," Journal of Management Science, vol. 31, no. 2, pp. 45-58, 2018. 
[75] W. J. Du, W. H. Han, and Q. Zhou, "Analysis on the obstacles in the formation and evolvement of technical standard alliances and the countermeasures," Science Research Management, vol. 31, no. 5, pp. 96-101, 2010.

[76] T. Fu and Y. A. Zhang, "A simulation study on evolution process of core-structure innovation network of industrial clusters: based on echo model," Journal of Systems \& Management, vol. 20, no. 4, pp. 406-415, 2011.

[77] J. Chen, H. W. Lu, and J. H. Dai, "Summarization of agentbased modeling and simulation," Computer Simulation, vol. 25, no. 12, pp. 1-7, 2008.

[78] U. Wilensky, Center for Connected Learning and ComputerBased Modeling, Northwestern University, Evanston, IL, USA, 1999, http://ccl.northwestern.edu/NetLogo/.

[79] I. Foster, Designing and Building Parallel Programs: Concepts and Tools for Parallel Software Engineering, Addison-Wesley, Boston, MA, USA, 1995.

[80] S. Y. Liao and J. H. Dai, "Study on complex adaptive system and agent-based modeling \& simulation," Journal of System Simulation, vol. 16, no. 1, pp. 113-117, 2004.

[81] V. K. Narayanan and T. Chen, "Research on technology standards: accomplishment and challenges," Research Policy, vol. 41, no. 8, pp. 1375-1406, 2012.

[82] J. Wen, W. J. Qualls, and D. Zeng, "Standardization alliance networks, standard-setting influence, and new product outcomes," Journal of Product Innovation Management, vol. 37, no. 2, pp. 138-157, 2020.

[83] J. Rodon, J. Ramis-Pujol, and E. Christiaanse, "A processstakeholder analysis of B2B industry standardisation," Journal of Enterprise Information Management, vol. 20, no. 1, pp. 83-95, 2007.

[84] D. P. Wang, W. Xiaoyan, and F. Fang, "The resource evolution of standard alliance by technology standardization," Chinese Management Studies, vol. 10, no. 4, pp. 787-801, 2016.

[85] R. Gulati, "Social structure and alliance formation patterns: a longitudinal analysis," Administrative Science Quarterly, vol. 40, no. 4, pp. 619-652, 1995.

[86] D. Lee, K. Kirkpatrick-Husk, and R. Madhavan, "Diversity in alliance portfolios and performance outcomes: a meta-analysis," Journal of Management, vol. 43, no. 5, pp. 1472-1497, 2017.

[87] Y. Li, H. Guo, S. Y. Cooper et al., "The influencing factors of the technology standard alliance collaborative innovation of emerging industry," Sustainability, vol. 11, no. 24, pp. 1-17, 2019. 